\title{
Expression and phosphorylation analysis of soluble proteins and membrane-localised receptor-like kinases from Arabidopsis thaliana in Escherichia coli
}

\author{
Eun-Seok Oh $\cdot$ Foyjunnaher Eva $\cdot$ Sang-Yun Kim $\cdot$ Man-Ho Oh
}

Received: 7 December 2018 / Revised: 13 December 2018 / Accepted: 13 December 2018

(C) Korean Society for Plant Biotechnology

\begin{abstract}
Molecular and functional characterization of proteins and their levels is of great interest in understanding the mechanism of diverse cellular processes. In this study, we report on the convenient Escherichia coli-based protein expression system that allows recombinant of soluble proteins expression and cytosolic domain of membrane-localised kinases, followed by the detection of autophosphorylation activity in protein kinases. This approach is applied to regulatory proteins of Arabidopsis thaliana, including 14-3-3, calmodulin, calcium-dependent protein kinase, TERMINAL FLOWER 1(TFL1), FLOWERING LOCUS T (FT), receptorlike cytoplasmic kinase and cytoplasmic domain of leucine-rich repeat-receptor like kinase proteins. Our Western blot analysis which uses phospho-specific antibodies showed that five putative LRR-RLKs and two putative RLCKs have autophosphorylation activity in vitro on threonine and/or tyrosine residue(s), suggesting their potential role in signal transduction pathways. Our findings were also discussed in the broader context of recombinant expression and biochemical analysis of soluble and membrane-localised receptor kinases in microbial systems.
\end{abstract}

Keywords Calcium-dependent protein kinase, Leucine-rich repeat receptor-like kinase(LRR-RLKs), Receptor-like cytoplasmic kinases (RLCKs), Post-translational modification, Arabidopsis thaliana, Escherichia coli

\section{Introduction}

Plants, as sessile organisms, are adapted to cope with adverse

E.-S. Oh $\cdot$ F. Eva $\cdot$ S.-Y. Kim $\cdot$ M.-H. Oh $(\bowtie)$

Department of Biological Sciences, College of Biological Sciences and Biotechnology, Chungnam National University, Daejeon 34134, Republic of Korea

e-mail:manhooh@cnu.ac.kr environmental conditions by employing complex signalling and control networks. Following stimuli perception by cells, signals are transmitted to downstream components by a variety of mechanisms, including activation of second messenger systems and post-translational modification, to elicit appropriate responses. Extensive research efforts over the past decades on functional analysis of various proteins have enriched our understanding of how plants respond to abiotic and biotic stresses, but many of their underlying mechanisms is still remain to be elucidated.

Protein phosphorylation is one of the most widespread post-translational modifications in both prokaryotes and eukaryotes. It is use as a regulatory signal by altering the conformational and dynamic state of the protein (Johnson and Barford, 1993). Phosphoproteome analysis of Arabidopsis thaliana revealed about 4000 phosphopeptides from all subcellular compartments, suggesting the involvement of protein phosphorylation in diverse cellular processes (Sugiyama et al. 2008; Reiland et al. 2009). In general, kinases and phosphatases regulate protein phosphorylation by reversibly catalysing the transfer of a phosphate group of ATP to hydroxyl groups of amino acid residues, predominantly on serine (Ser), threonine (Thr) and tyrosine (Tyr) residues.

In eukaryotes, protein kinases are divide into three families: Tyr kinase, Ser/Thr kinase and Ser/Thr/Tyr kinase (also termed dual-specificity kinase) (Lindberg et al. 1992). Unlike animals, plants have no genes encoding classical Tyr kinase homologs, and only about $4 \%$ of plant phosphopeptides are Tyr-phosphorylated (Sugiyama et al. 2008; Rudrabhatla et al. 2006). Nevertheless, there is accumulating evidence that Tyr phosphorylation in plants contributes to the regulation of various pathways, including gibberellin response, brassinosteroid signalling, innate immunity and root nodule symbiosis (Macho et al. 2014; Lin et al. 2014; Oh et al. 2009; Nemoto et al. 2017; Saha et al. 2016). To date, several members of kinase, including 
calcium-dependent protein kinase (CPK), leucine-rich repeat (LRR)-receptor-like kinase (RLK), receptor-like cytoplasmic kinase (RLCK) and mitogen-activated protein kinase (MAPK), have been reported to be responsible for Tyr phosphorylation signalling in plants by acting as the dual-specificity kinase, and kinase themselves, 14-3-3 proteins and ubiquitin ligases have been found as target substrates (Rameneni et al. 2015; Nemoto et al. 2017; Saha et al. 2016; Matsuoka et al. 2018; Swatek et al. 2014; Oh et al. 2012).

In Arabidopsis, RLKs belong to a monophyletic gene family, containing over 600 members, which represent about $60 \%$ of all kinase proteins, and generally consist of an extracellular domain, a transmembrane domain and a cytoplasmic domain (CD) (Shiu and Bleecker, 2003). The group of LRRRLKs is the largest RLK subfamily with more than 200 members and only found in plants (Shiu and Bleecker 2003; Hwang et al. 2011). LRR motif on the ectodomain mediates protein-protein interaction via its unique repeat sequences, allowing the perception of various external ligands, including hormone, pathogen molecules and small peptides (Kobe and Deisenhofer, 1994; Shiu et al. 2004). The CD is composed of a juxtamembrane domain, kinase domain and carboxyl-terminal domain. In BRI1, the LRR-RLK involved in brassinosteroid signalling, phosphorylation at the juxtamembrane domain and carboxy-terminal domain residues is required for oligomerization of RLKs and/or signal transduction to downstream components, indicating the regulation of RLKs as a multimeric complex (Wang et al. 2008; Wang et al. 2005). On the other hand, about $25 \%$ of RLKs, called RLCKs, carry no extracellular and transmembrane domains (Jurca et al. 2008). Many RLCKs, including BSK1, PBS1 and BIK1, are still closely associated with membrane-localised RLKs and play a role in intracellular signal transduction of plant development and immunity (Tang et al. 2008; Lu et al. 2010; Shao et al. 2003; Zhang et al. 2010).

Given the important role of phosphorylation in regulating cellular processes, sensitive and specific approaches for detecting protein phosphorylation are critical for understanding signal transduction pathways. Herein, we employed an Escherichia coli protein expression system to investigate signalling networks between substrates and receptor kinases in Arabidopsis. We successfully produced a soluble form of various regulatory proteins, including 14-3-3, calmodulin (CaM), CPK, TERMINAL FLOWER 1(TFL1), FLOWERING LOCUS T (FT), the CD of LRR-RLK and RLCK proteins, some of which were further analysed by immunoblots with phospho-specific antibodies to determine autophosphorylation activities in vitro. We identified five putative LRR-RLKs and two putative RLCKs that might play a role in signal transduction through phosphorylation at threonine and/or tyrosine residue(s). This approach is useful for not only the identification of specific post-translational modification but also for the characterization of the regulatory role at a given modification.

\section{Materials and methods}

Recombinant protein expression and immunopurification

To produce recombinant proteins, cDNA sequences of Arabidopsis 14-3-3s, CaMs, AtCPK4, FT, and TFL1 were cloned into the pET15b vector (His-tag) for soluble protein expression, and those of the CD of the LRR-RLKs and RLCKs were cloned into the Gateway-modified pFlag-Mac vector (Flag-tag). The resulting constructs were introduced into $E$. coli BL21 (DE3) cells (Novagen, Rockland, MA, USA), and the transformants were grown in LB broth with ampicillin at $50 \mu \mathrm{g} / \mathrm{ml}$. When the cell culture reached the optical density $\left(\mathrm{OD}_{600}\right) 0.6$, protein expression was induced with $300 \mu \mathrm{M}$ isopropyl $\beta$-D-1-thiogalactopyranoside (IPTG) for $16 \mathrm{~h}$ at room temperature. Expression and purification of recombinant His- and Flag-tagged proteins was perform according to previously described methods (Chae et al. 2016). After elution of the recombinant proteins from beads, protein solutions were dialysed against a $1000 \times$ volume of buffer containing $20 \mathrm{mM} 3$-(N-morpholino) propanesulfonic acid (MOPS) (pH 7.5) and $1 \mathrm{mM}$ DTT, as previously described (Oh et al. 2000). After dialysis of purified recombinant proteins, protein concentration was measure by the Bradford assay.

\section{Electrophoresis and Western blot analysis}

All immunopurified recombinant proteins were prepared according to previously described methods (Oh et al. 2009; Oh et al. 2018). Polyvinylidene fluoride (PVDF) membranes were probe with anti-Flag, pThr polyclonal antibodies and pTyr (pY) monoclonal antibodies that recognise phosphorylated threonine and tyrosine residues. Alternatively, PVDF membranes were stain with ProQ Diamond reagent (Invitrogen, Grand Island, NY, USA). After incubation with specific primary antibodies for $6 \mathrm{~h}$ at room temperature, membranes were washed three times with PBST for 5 min each time and incubated with an Alexa Fluor 680-conjugated secondary antibody (Thermo Fisher Scientific, Rockford, IL, USA) diluted at 1:10,000 in PBST. Western blot hybridisation images were obtain using a LI-COR C-DIGIT scanner (LI-COR Biosciences, Lincoln, NE, USA). 


\section{Results and Discussion}

In biochemical analysis of protein of interest, production of recombinant proteins should be proceeded to fully determining its kinetics and post-translational modifications. Since obtaining enough amounts of proteins in a functionally active form is critical, diverse expression platforms have been developed and studied. Among them, E. coli is one of the most popular expression platforms by offering several advantages: its culture reaches high cell density in relatively simple growth condition, and a number of vectors that allow for controlling the rate of protein expression have been well established (Rosano and Ceccarelli, 2014). In this study, using E. coli BL21 (DE3) strain and two different protein expression vectors, $\mathrm{pET} 15 \mathrm{~b}$ and $\mathrm{pFlag}-\mathrm{Mac}, A$. thaliana regulatory proteins, including cytosolic proteins (14-3-3s, CaMs, AtCPK4, TFL1 and FT) and cytoplasmic domain of membrane proteins (LRR-RLKs and RLCKs), were purified and analysed by Western blot to determine protein expression levels and posttranslational modifications.

The 14-3-3 proteins are a family of highly conserved, soluble, acidic proteins found in all eukaryotes (Fu et al. 2000). They specifically bind to phosphoserine and/or phosphothreonine residues of target proteins and mediate diverse signalling pathways, including cell metabolism, hormone signalling, protein trafficking, abiotic stress tolerance and defence response (Yaffe and Smerdon, 2001). Among multiple isoforms of 14-3-3 proteins in Arabidopsis (Chae et al. 2016), we cloned cDNA sequence of kappa and lambda 14-3-3 isoforms into pET15b, which carries an N-terminal His-tag sequence, and introduced the expression vector into $E$. coli BL21(DE3) strain. His $_{6}$-tagged 14-3-3 proteins were immunopurified using Ni-NTA resin and detected by Coomassie Brilliant Blue staining, following separation by SDS-PAGE (Fig. 1A).

In plants, transient fluctuation of $\mathrm{Ca}^{2+}$ concentration inside cells is widely observed upon exposure to stress conditions, suggesting that $\mathrm{Ca}^{2+}$ plays an important role in cell signalling networks (Virdi et al. 2015). There are several known families of $\mathrm{Ca}^{2+}$ sensory proteins, including $\mathrm{CaM}$ and $\mathrm{CPK} . \mathrm{Ca}^{2+}$ binds to $\mathrm{CaM}$ via EF-hand motifs and triggers conformational changes, leading to interaction with the target proteins (Babu et al. 1988; DeFalco et al. 2010). On the other hand, since CPK carries both EF-hand motif and kinase domain, it can directly respond to $\mathrm{Ca}^{2+}$ influx without interaction with $\mathrm{CaM}$ (Cheng et al. 2002). We selected two isoforms of $\mathrm{CaM}(\mathrm{CaM} 2$ and CaM7) and one isoform of CPK (AtCPK4) in Arabidopsis for the production of recombinant proteins. Their cDNA sequences were cloned into the pET15b, expressed in E. coli BL21 (DE3) strain and purified using Ni-NTA resin. All the three His $_{6}$-tagged proteins were isolated in the soluble

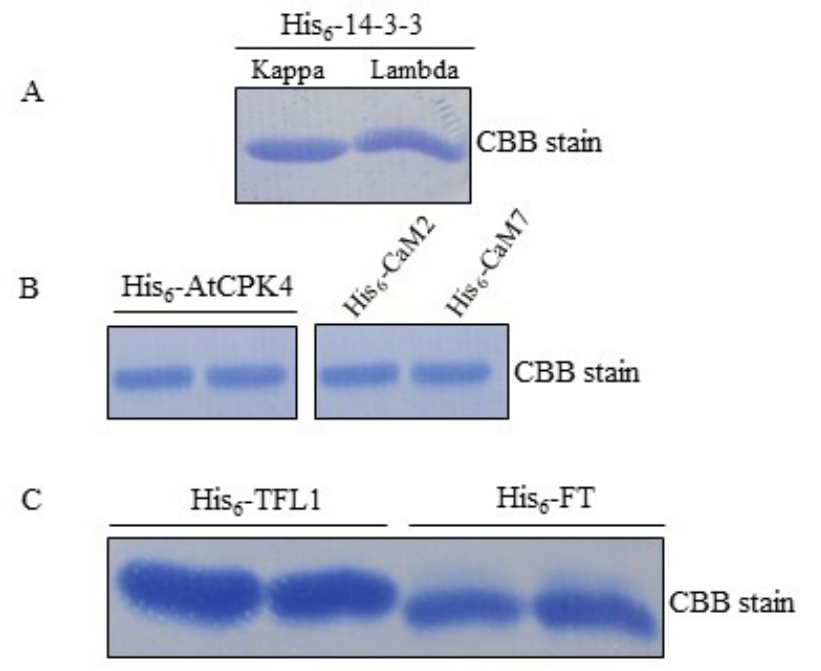

Fig. 1 Recombinant protein production using the Escherichia coli expression system. Coomassie Brilliant Blue (CBB) R-250 staining clearly showing the successful recombinant expression of (A) 14-3-3s, (B) AtCPK4, CaM2, CaM7, (C) TFL1 and FT. Protein expression induced by adding IPTG to the cell culture for 16 hours at room temperature. Total crude extracts were affinity purified using Ni-NTA resin, separated using 12\% SDS-PAGE and stained with CBB

fraction and confirmed by SDS-PAGE (Fig. 1B).

FT and TFL1 proteins of Arabidopsis were also obtain successfully in a similar way using $E$. coli expression platform (Fig. 1C). Both of them are members of phosphatidyl ethanolamine-binding protein (PEBP) family that regulates the activity of diverse enzymes by functioning as a scaffold for the interaction between proteins (Pnueli et al. 2001; Schoentgen et al. 1987). Although FT and TFL1 share a similar amino acid sequence identity with about $60 \%$, FT and TFL1 act antagonistically in the regulation of flowering-time genes (Hanzawa et al. 2005; Wickland and Hanzawa, 2015). However, much remains to be revealed about molecular mechanisms of not only FT/TFL1, but also 14-3-3, CaM and CPK. Isolating soluble forms of these proteins could serve as the first step to further elucidate the structural and functional properties.

In addition to cytosolic proteins, the $\mathrm{CD}$ expression of plasma membrane-localised LRR-RLKs was also test in the E. coli system (Park et al. 2015). Plant LRR-RLKs are involved in the tight control of growth, morphogenesis, pathogen resistance and abiotic stress responses (Becraft, 2002; De Lorenzo et al. 2009; Tör et al. 2009; Torii et al. 2004). Among three domains of the $\mathrm{CD}$, kinase domain is mainly responsible for phosphoryl transfer from ATP to amino acid residues or other protein substrates of downstream components. However, the carboxyl-terminus region of receptor kinases, carrying juxtamembrane domain and carboxyl-terminal domain, often plays an important regulatory role, which might be modulate 
A

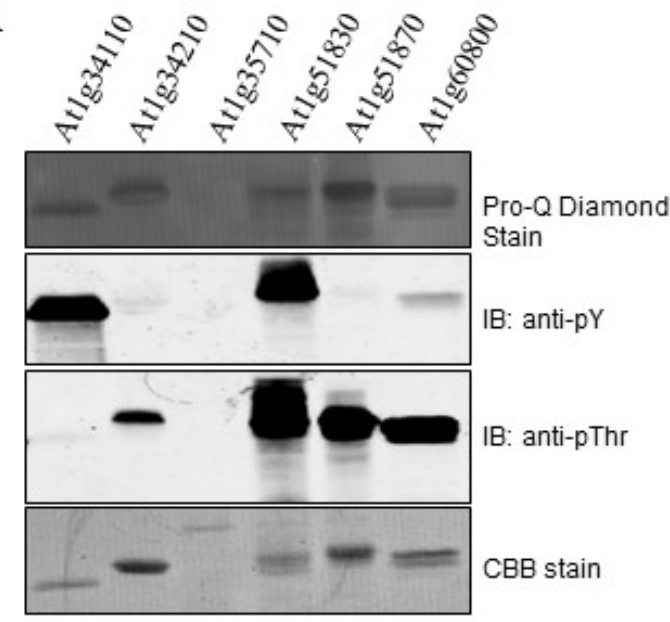

B

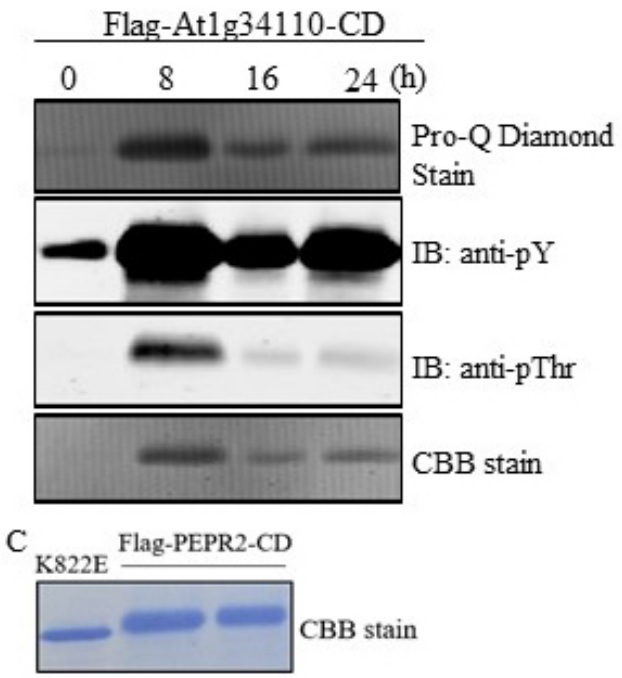

Fig. 2 Production and phospho-analysis of cytoplasmic domain (CD) of LRR-RLKs in Escherichia coli expression platform. (A) Analysis of CD of six different Flag-tagged LRR-RLKs (At1g34110, At1g34210, At1g35710, At1g51830, At1g51870, At1g60800) at single times following addition of IPTG (B) Analysis of Flag-At1g34100-CD phosphoprotein production at different induction times after addition of IPTG. For immunoblot analysis, PVDF membranes were probed with phosphothreonine and phosphotyrosine-specific antibodies

by the phosphorylation of serine, threonine, and tyrosine residues (Oh et al. 2018; Bentem and Hirt, 2009).

In the present study, we cloned cDNA sequences of LRRRLK CDs into the pFlag-Mac expression vector, introduced them into E. coli BL21 (DE3) strain and performed comparative biochemical assays. The phosphorylation levels of the expressed recombinant proteins were monitored at different time points after induction using anti-phosphothreonine (pThr) and anti-phosphotyrosine (pTyr) antibodies along with ProQ Diamond stain (Fig. 2A, B). Among six different LRRRLKs tested, four LRR-RLKs (At1g34210, At1g51830, At1g 51870 and At1g60800) were autophosphorylated on threonine residue(s) and three LRR-RLKs (At1g34110, At1g51830 and At1g60800) were autophosphorylated on tyrosine residue(s). No phosphorylation on threonine and tyrosine residue(s) was observe in At1g35710. Interestingly, At1g51830 showed strong autophosphorylation on both threonine and tyrosine residue(s), suggesting the presence of dual-specificity kinase activity (Fig. 2A). Similar observations of autophosphorylation activity in $E$. coli have been also report for CDs of Arabidopsis BRI1 and BAK1 (Oh et al. 2009; Oh et al. 2018). We also found that the level of At 1934110 autophosphorylation on threonine and tyrosine residue(s) was change at different induction times (Fig. 2B). In particular, phoshporylation at threonine residue(s) reached its peak intensity at $8 \mathrm{~h}$ after induction, but was barely detect at other time points. These autohosphorylation and transphosphorylation activities on tyrosine residues have been reported in many plant receptor kinases, including PRK1, AtSERK1 and light-repressible kinases, suggesting that At1g34110 receptor kinase might play an important role in cell signaling pathway as upstream component in Arabidopsis thaliana.

RLCK proteins, belonging to RLK superfamily, lack extracellular and transmembrane regions. Many studies have revealed that plant RLCKs play a central role in defense response by activating signal transduction between PRR complexes and MAPK (Couto and Zipfel 2016). In addition to kinase domain, RLCKs contain N- and C-terminal extensions possibly implicated in association with membrane-bound RLKs or for regulatory purposes via post-translational modification (Antol ín-Llovera et al. 2012). Although few RLCKs, such as BSK1, PBS1 and BIK1, have been well-characterize, most RLCKs remain to be explore at the molecular level.

Here, we tested eight Arabidopsis RLCKs for autophosphorylation kinase activities in vitro using E. coli system (Fig. 3). We cloned full-length cDNA sequences of Arabidopsis RLCKs into the pFlag-Mac expression vector, introduced them into $E$. coli BL21 (DE3) strain and analysed by immunoblots with phospho-specific antibodies. Among eight RLCKs tested, only two RLCKs (At1g06700 and At4g13190) had distinct autophosphorylation activity in E. coli. Immunoblots of At1g06700 showed autophosphorylation at tyrosine and threonine residue(s), indicating multi-specific kinase activity, while that of At4g13190 showed autophosphorylation only at threonine residue(s). Given that phosphorylation of RLKs generally initiates and mediates signal transduction pathways, 


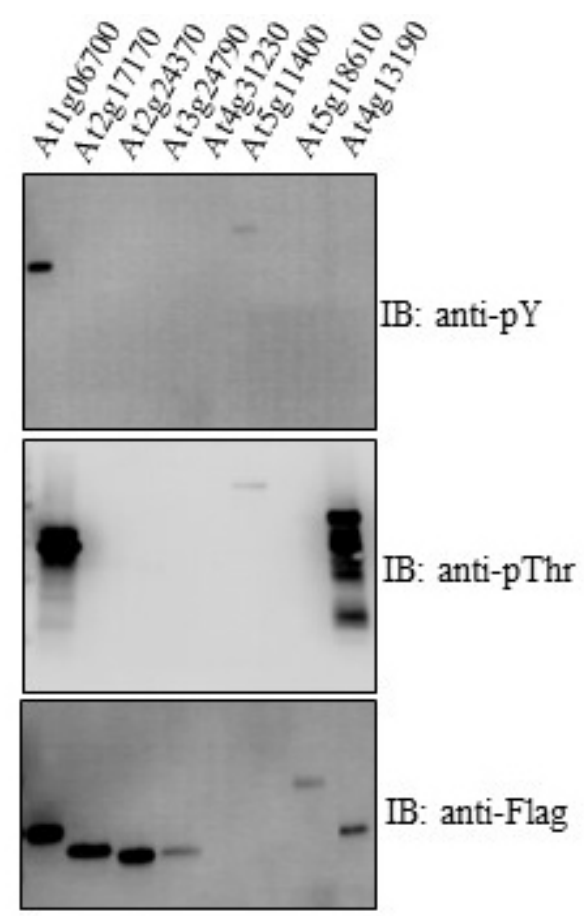

Fig. 3 Autophosphorylation of recombinant Arabidopsis RLCK proteins expressed in Escherichia coli. Anti-phosphothreonine and anti-phosphotyrosine immunoblots showing patterns of autophosphorylation. Not all RLCKs were capable of autophosphorylation following induction in E. coli. Autophosphorylation activity of recombinant proteins was monitored 16 hours after induction of receptor kinase expression. Each sample showed total crude proteins, including FLAG-tagged receptor kinase, extracted from $E$. coli and subjected to $10 \%$ sodium dodecyl sulfate polyacrylamide gel electrophoresis (SDS-PAGE) before transfer to polyvinylidene difluoride (PVDF) membranes. Membranes were immunoblotted (IB) using the antibodies indicated

these two RLCKs might be also involved in sensing and responding to cellular needs in Arabidopsis.

Overall, our results demonstrated the successful expression and immunopurification of recombinant proteins combined with subsequent protein phosphorylation analysis using $E$. coli in vitro system. It is applicable for both soluble proteins and membrane-localised receptor kinases, and can be employ as a useful tool for studying molecular mechanisms and functions of protein of interest. Importantly, our assays for autophoshporylation of receptor kinases could be apply more broadly to a wide range of recombinant proteins, and provides a convenient and powerful system for elucidating kinase specificity in vitro.

\section{Acknowledgements}

This work was supported by a grant from Chungnam National University (CNU).

\section{Conflicts of Interest}

The authors declare no conflict of interest.

\section{References}

Antoln-Llovera M, Ried MK, Binder A, Parniske M (2012) Receptor kinase signaling pathways in plant-microbe interactions. Annu. Rev. Phytopathol 50:451-473

Babu YS, Bugg CE, Cook,WJ. (1988) Structure of calmodulin refined at $2.2 \AA$ resolution. Journal of molecular biology 204: 191-204

Becraft PW. (2002) Receptor kinase signaling in plant development. Annu. Rev. Cell Dev. Biol 18:163-192

Bentem SF, Hirt H. (2009) Protein tyrosine phosphorylation in plants: more abundant than expected?. Trends in Plant Science 14:71-76

Chae WB, Park Y-J, Lee KS, Nou I-S, Oh M-H (2016) Plant receptor kinases bind and phosphorylate 14-3-3 proteins. Genes and Genomics 38:1111-1119

Cheng SH, Willmann MR, Chen HC, Sheen J (2002) Calcium signaling through protein kinases. The Arabidopsis calciumdependent protein kinase gene family. Plant physiology 129: 469-485

Couto D, Zipfel C (2016) Regulation of pattern recognition receptor signalling in plants. Nature Reviews Immunology 16:537-552

DeFalco TA, Bender KW, Snedden WA (2010) Breaking the code: $\mathrm{Ca} 2+$ sensors in plant signalling. Biochemical Journal 425 : $27-40$

De Lorenzo L, Merchan F, Laporte P, Thompson R, Clarke J, Sousa C, Crespi, M (2009) A novel plant leucine-rich repeat receptor kinase regulates the response of Medicago truncatula roots to salt stress. Plant Cell 21:668-680

Fu H, Subramanian RR, Masters SC (2000). 14-3-3 proteins: structure, function, and regulation. Annual review of pharmacology and toxicology 40:617-647

Hanzawa Y, Money T, Bradley D (2005) A single amino acid converts a repressor to an activator of flowering. Proceedings of the National Academy of Sciences 102:7748-7753

Hwang SG, Kim DS, Jang CS (2011) Comparative analysis of evolutionary dynamics of genes encoding leucine-rich repeat receptor-like kinase between rice and Arabidopsis. Genetica 139:1023-1032

Rameneni JJ, Lee Y, Dhandapani V, Yu X, Choi SR, Oh MH, Lim YP (2015) Genomic and post-translational modification analysis of Leucine-Rich-Repeat Receptor-Like Kinases in Brassica rapa. PLoS ONE DOI:10.1371/journal.pone.0142255

Jurca ME, Bottka S, Fehér A (2008) Characterization of a family of Arabidopsis receptor-like cytoplasmic kinases (RLCK class VI). Plant cell reports 27:739-748

Johnson LN, Barford D. (1993) The effects of phosphorylation on the structure and function of proteins. Annu. Rev. Bioph. 
Biom 22:199-232

Kobe B, Deisenhofer J. (1994) The leucine-rich repeat: a versatile binding motif. Trends in Biochemical Sciences 19:415-421

Lindberg RA, Quinn AM, Hunter T. (1992) Dual-specificity protein kinases: will any hydroxyl do?. Trends in Biochemical Sciences 17:114-119

Lin W, Li B, Lu D, Chen S, Zhu N, He P, Shan L. (2014) Tyrosine phosphorylation of protein kinase complex BAK1/BIK1 mediates Arabidopsis innate immunity. Proceedings of the National Academy of Sciences 111:3632-3637

Lu D, Wu S, Gao X, Zhang Y, Shan L, He P. (2010) A receptor-like cytoplasmic kinase, BIK1, associates with a flagellin receptor complex to initiate plant innate immunity. Proceedings of the National Academy of Sciences 107:496-501

Macho AP, Schwessinger B, Ntoukakis V, Brutus A, Segonzac C, Roy S, Kadota Y, Oh MH, Sklenar J, Derbyshire P, LozanoDurán R, Malinovsky FG, Monaghan J, Menke FL, Huber SC, He SY, Zipfel C (2014) A bacterial tyrosine phosphatase inhibits plant pattern recognition receptor activation. Science 343:1509-1512

Matsuoka D, Furuya T, Iwasaki T, Nanmori T. (2018) Identification of tyrosine autophosphorylation sites of Arabidopsis MEKK 1 and their involvement in the regulation of kinase activity. FEBS letters 592:3327-3334

Nemoto K, Ramadan A, Arimura GI, Imai K, Tomii K, Shinozaki K, Sawasaki T (2017) Tyrosine phosphorylation of the GARU E3 ubiquitin ligase promotes gibberellin signalling by preventing GID1 degradation. Nature Communications 8:1004

Oh MH, Wu X, Kim HS, Harper JF, Zielinski RE, Clouse SD, Huber SC (2012) CDPKs are dual-specificity protein kinases and tyrosine autophosphorylation attenuates kinase activity. FEBS letters 586:4070-4075

Oh M-H, Ray WK, Huber SC, Asara JM, Gage DA, Clouse SD. (2000) Recombinant brassinosteroid insensitive 1 receptor-like kinase autophosphorylates on serine and threonine residues and phosphorylates a conserved peptide motif in vitro. Plant Physiol 124:751-766

Oh MH, Wang X, Kota U, Goshe MB, Clouse SD, Huber SC. (2009) Tyrosine phosphorylation of the BRI1 receptor kinase emerges as a component of brassinosteroid signaling in Arabidopsis. Proceedings of the National Academy of Sciences 106:658-663

Oh E-S, Lee Y, Chae W, Rameneni J, Park Y-S, Lim Y, Oh M-H. (2018) Biochemical analysis of the role of Leucine-Rich Repeat Receptor-Like kinases and the carboxy-terminus of receptor kinases in regulating kinase activity in Arabidopsis thaliana and Brassica oleracea. Molecules doi: 10.3390/ molecules23010236

Park, Y.-L., Yang, H.-S., Oh, M.-H. Tyrosine phosphorylation as signaling component for plant improvement. Journal of Plant Biotechnology 2015, 42:277-283

Pnueli L, Gutfinger T, Hareven D, Ben-Naim O, Ron N, Adir N, Lifschitz E (2001) Tomato SP-interacting proteins define a conserved signaling system that regulates shoot architecture and flowering. Plant Cell 13:2687-2702
Reiland S, Messerli G, Baerenfaller K, Gerrits B, Endler A, Grossmann J, Gruissem W, Baginsky S (2009) Large-scale Arabidopsis phosphoproteome profiling reveals novel chloroplast kinase substrates and phosphorylation networks. Plant Physiol 150:889-903

Rosano G L, Ceccarelli EA (2014) Recombinant protein expression in Escherichia coli: advances and challenges. Frontiers in microbiology doi: 10.3389/fmicb.2014.00172

Rudrabhatla P, Reddy, MM, Rajasekharan R (2006) Genome-wide analysis and experimentation of plant serine/threonine/ tyrosine-specific protein kinases. Plant Molecular Biology 60:293-319

Saha S, Paul A, Herring L, Dutta A, Bhattacharya A, Samaddar S, Goshe MB, DasGupta, M (2016) Gatekeeper Tyrosine phosphorylation of SYMRK is essential for synchronising the epidermal and cortical responses in root nodule symbiosis. Plant Physiology 171:71-81

Schoentgen, F, Saccoccio F, Jolles J, Bernier I, Jolles P (1987) Complete amino acid sequence of a basic $21-\mathrm{kDa}$ protein from bovine brain cytosol. European Journal of Biochemistry 166: 333-338

Shiu SH, Bleecker AB (2003) Expansion of the receptor-like kinase/Pelle gene family and receptor-like proteins in Arabidopsis. Plant Physiology 132: 530-543

Shao F, Golstein C, Ade J, Stoutemyer M, Dixon JE, Innes RW (2003) Cleavage of Arabidopsis PBS1 by a bacterial type III effector. Science 301:1230-1233

Shiu SH, Karlowski WM, Pan R, Tzeng YH, Mayer KF, Li, WH (2004) Comparative analysis of the receptor-like kinase family in Arabidopsis and rice. The Plant Cell 16:1220-1234

Sugiyama N, Nakagami H, Mochida K, Daudi A, Tomita M, Shirasu K, Ishihama Y (2008) Large-scale phosphorylation mapping reveals the extent of tyrosine phosphorylation in Arabidopsis. Mol Syst Biol 4:193

Swatek KN, Wilson RS, Ahsan N, Tritz RL, Thelen JJ (2014) Multisite phosphorylation of 14-3-3 proteins by calciumdependent protein kinases. Biochemical Journal 459:15-25

Tang W, Kim TW, Oses-Prieto JA, Sun Y, Deng Z, Zhu S, Wang R, Burlingame AL, Wang ZY (2008) BSKs mediate signal transduction from the receptor kinase BRI1 in Arabidopsis. Science 321:557-560

Tör M, Lotze MT, Holton N (2009) Receptor-mediated signalling in plants: Molecular patterns and programmes. J Exp Bot 60: 3645-3654

Torii S, Kusakabe M, Yamamoto T, Maekawa M, Nishida E. (2004) SEF is a spatial regulator for Ras/MAP kinase signaling. Dev Cell 7:33-44

Virdi AS, Singh S, Singh P (2015) Abiotic stress responses in plants: roles of calmodulin-regulated proteins. Frontiers in Plant Science 6:809

Wang X, Li X, Meisenhelder J, Hunter T, Yoshida S, Asami T, Chory J (2008) Autoregulation and homodimerization are involved in the activation of the plant steroid receptor BRI1. Developmental Cell 8:855-865

Wang X, Goshe MB, Soderblom EJ, Phinney BS, Kuchar JA, Li J, 
Asami T, Yoshida S, Huber, SC, Clouse SD. (2005) Identification and functional analysis of in vivo phosphorylation sites of the Arabidopsis BRASSINOSTEROID-INSENSITIVE1 receptor kinase. The Plant Cell 17:1685-1703

Wickland DP, Hanzawa Y. (2015) The FLOWERING LOCUS T/TERMINAL FLOWER 1 gene family: functional evolution and molecular mechanisms. Molecular Plant 8:983-997

Zhang J, Li W, Xiang T, Liu Z, Laluk K, Ding X, Zou Y, Gao M,
Zhang X, Chen S, Mengiste T, Zhang Y, Zhou JM (2010) Receptor-like cytoplasmic kinases integrate signaling from multiple plant immune receptors and are targeted by a Pseudomonas syringae effector. Cell Host Microbe 7: 290-301

Yaffe MB, Smerdon SJ (2001) PhosphoSerine/threonine binding domains: you can't pSERious? Structure 9: R33-R38 